\title{
Real-Time Distributed Control for Smart Electric Vehicle Chargers: From a Static to a Dynamic Study
}

\author{
Omid Ardakanian, Student Member, IEEE, Srinivasan Keshav, Member, IEEE, and \\ Catherine Rosenberg, Fellow, IEEE
}

\begin{abstract}
At high penetrations, uncontrolled electric vehicle (EV) charging has the potential to cause line and transformer congestion in the distribution network. Instead of upgrading components to higher nameplate ratings, we investigate the use of real-time control to limit EV load to the available capacity in the network. Inspired by rate control algorithms in computer networks such as TCP, we design a measurement-based, real-time, distributed, stable, efficient, and fair charging algorithm using the dual-decomposition approach. We show through extensive numerical simulations and power flow analysis on a test distribution network that this algorithm operates successfully in both static and dynamic settings, despite changes in home loads and the number of connected EVs. We find that our algorithm rapidly converges from large disturbances to a stable operating point. We show that in a test setting, for an acceptable level of overload, only 70 EVs could be fully charged without control, whereas up to around $700 \mathrm{EVs}$ can be fully charged using our control algorithm. This compares well with the maximum supportable population of approximately 900 EVs. Our work also provides engineering guidelines for choosing the control parameters and setpoints in a distribution network.
\end{abstract}

Index Terms-Distributed algorithms, electric vehicles, optimal control.

\section{INTRODUCTION}

A T HIGH penetration levels, uncontrolled electric vehicle charging can congest lines and transformers and cause voltage swings in the distribution system [1], [2]. Even at low penetration levels, uncontrolled charging can lead to congestion in certain neighborhoods, due to a non-homogeneous distribution of EVs in the distribution network. Unrelieved congestion can overheat transformer windings and accelerate degradation of line and transformer insulation, leading to premature equipment failure. Although distribution system congestion can be relieved by upgrading system components piecemeal, this approach is both time-consuming and expensive. A more cost-effective alternative is for utility companies to directly control

Manuscript received August 14, 2013; revised December 09, 2013, February 07, 2014, and April 07, 2014; accepted May 25, 2014. Date of publication August 05, 2014; date of current version September 05, 2014. Paper no. TSG-00661-2013.

O. Ardakanian and S. Keshav are with the Cheriton School of Computer Science, University of Waterloo, Waterloo, ON N2L 3G1, Canada (e-mail: oardakan@uwaterloo.ca; keshav@uwaterloo.ca).

C. Rosenberg is with the Department of Electrical and Computer Engineering, University of Waterloo, Waterloo, ON N2L 3G1, Canada (e-mail: cath@uwaterloo.ca).

Color versions of one or more of the figures in this paper are available online at http://ieeexplore.ieee.org.

Digital Object Identifier 10.1109/TSG.2014.2327203 smart EV chargers ${ }^{1}$ so that system components are rarely overloaded. This is the motivation for our work.

In prior work, EV chargers have been controlled using a schedule computed either the prior day (known as pre-dispatch scheduling) [1], [3]-[7] or in real-time [8]-[12]. Pre-dispatch scheduling approaches typically compute the charging schedule by solving a power flow problem. This requires precise estimates of non-EV loads, the points of connection of active EVs, their arrival and departure times, and the initial state of charge of their batteries. These parameters are difficult to predict accurately. Therefore, these approaches maintain a conservative operating margin to accommodate estimation uncertainties, which under-utilizes system resources.

In contrast, the real-time computation of charging schedules, which is the focus of this paper, achieves higher utilization by continuously adapting the charging rate of EV chargers to the measured available capacity of the network. In this approach, enabled by the widespread adoption of measurement and communication infrastructure in future distribution systems, line current and voltage measurements are sent from measurement nodes to control elements, and the control signals from these control elements are sent to EV chargers [13]. This allows EV chargers to use higher rates when there is available capacity, reducing these rates when the distribution network becomes congested. Note that during demand peaks, the available capacity of the network may not allow all EV chargers to charge at their maximum rate. Therefore, it is desirable to allocate the available capacity fairly among EV chargers. Thus, computing the set of EV charging rates can be viewed as an optimization problem whose solution is an allocation that simultaneously satisfies efficiency and fairness criteria.

Drawing on the design of congestion control protocols in packet-switched networks [14]-[17], our prior work [12] formulates a nonlinear convex optimization problem to obtain a charging rate allocation which is both proportionally fair [18], and scale-invariant Pareto optimal [19]. Solving the optimization problem allows us to obtain control rules that are implemented using dual decomposition [20] and the projected subgradient method [21]. In other words, we decompose the dual optimization problem into several subproblems, each solved independently and iteratively by an EV charger to adjust its charging rate. These subproblems are coordinated by a master problem through congestion prices [18], which are computed based on the congestion state of distribution lines and transformers and are periodically sent to EV chargers. We refer to this approach as distributed control.

\footnotetext{
${ }^{1} \mathrm{~A}$ smart EV charger chooses a charging rate that not only optimizes battery life but also is responsive to control signals it receives.
} 
Our prior work studied a quasi-static setting, where home loads were assumed to not change between snapshots. In this paper, we study our distributed control scheme in a dynamic setting, where home loads and the number of EVs being charged change over time. We validate using power flow analysis on a standard test distribution system that our control algorithm does not violate the operational limits of the distribution network. We investigate sensitivity of our control algorithm to arrivals and departures of EVs, the EV penetration level, the rated charge capacity of EV chargers, the choice of control parameters, and control setpoints. We make the following three contributions:

- We present a TCP-inspired measurement-based distributed control of EV charging and analyze its worst-case convergence.

- Using synthetic household load traces and an accurate power flow simulator, we numerically evaluate our control algorithm in a dynamic setting using extensive simulations, and study the performance sensitivity to the choice of control parameters.

- We provide engineering insights into the dynamic operation of the real-time distributed algorithm and discuss different design choices for control parameters to meet utility performance requirements.

This paper extends our prior work [12] in three ways. First, we validate the operation of the distributed control algorithm in a dynamic setting using power flow analysis, rather than in a quasi-static setting, as we had done earlier. Second, we analyze the convergence speed of the algorithm in the worst case. Finally, we provide several engineering insights.

\section{RELATED WORK}

The potential impacts of introducing EVs into the distribution network have been explored extensively in the literature and many scheduling algorithms have been proposed to control EV charging load. Most existing work proposes centralized control of EV chargers. However, as discussed in a recent white paper [22], coordinating control at different levels becomes infeasible with such centralized control. This makes distributed control of EV charging and other responsive loads the better approach. Moreover, distributed control algorithms scale well with the size of the network and the number of EV chargers, and are robust to failure of a single measurement or control node. Therefore, we only review distributed control algorithms in the remainder of this section.

In recent work, Gan et al. [7] and Ma et al. [6] use distributed control to obtain a day-ahead charging schedule for EVs. Gan et al. formulate the EV charging control problem as an optimization problem with the objective of flattening the aggregate demand served by a transformer. A stochastic distributed control algorithm is proposed to find an approximate solution to this optimization problem. It is shown that this algorithm almost surely converges to one of the equilibrium charging profiles. In [6], a decentralized algorithm is proposed to find the EV charging strategy that minimizes individual charging costs. It is shown that the optimal strategy obtained using this algorithm converges to the unique Nash equilibrium strategy when there is an infinite population of EVs. In the case of homogeneous EV populations, this Nash equilibrium strategy coincides with the valley-filling maximizing strategy (i.e., the globally optimal strategy).
Our approach differs from these two approaches in two ways. First, their goal is to simply flatten the load served by the substation transformer, whereas we deal with line and transformer overloading in the entire distribution network. Second, these algorithms do not guarantee fair allocation of the available network capacity to EVs; this is an important property of our control mechanism.

Turning our attention to distributed real-time control, the closest line of work to ours is by Wen et al. [10], which selects a subset of connected EVs for charging at every timeslot so as to maximize user convenience subject to circuit-level demand constraints. This is formulated as a combinatorial optimization problem, and a centralized algorithm is proposed to solve a convex relaxation of this problem. Furthermore, a distributed algorithm is proposed to solve the optimization problem using the alternating direction method of multipliers for distributed optimization. Despite this similarity in using distributed optimization to schedule EV chargers in real-time, our approach differs in three ways. First, their focus is on satisfying user-specified charging requirements such as the charging deadline and the final state of charge, while our main goal is to balance efficiency and fairness. ${ }^{2}$ Second, their formulation reflects only constraints imposed by two layers of a distribution network, whereas we model the entire distribution network, taking into account the capacities of all lines and transformers. Finally, unlike this work, they do not analyze the performance sensitivity of their control algorithm to the choice of control setpoints.

A recent paper by Fan [11] borrows the notion of congestion pricing from the Internet to reduce the peak load while providing weighted proportional fairness to end users. Exploiting the two way communications between the utility and users, congestion prices are sent to users, enabling them to adapt their demands to the capacity of the market in a fully distributed fashion. The user preference is modelled as a willingness to pay parameter, i.e., the weight factor in the utility function of users. The proposed algorithm is then applied to EV charging to obtain a charging rate allocation. Interestingly, the total EV charging load varies with the range from which the weight factors can be chosen. Thus, the utility has to limit this range to ensure that the total load is not greater than the market capacity. The paper also studies the convergence behavior of the algorithm using both analysis and simulation results. However, unlike our approach, this paper does not model the distribution network and does not incorporate the capacity constraints of distribution lines and transformers, and the charging rate constraints of EV chargers.

In [9], additive-increase-multiplicative-decrease (AIMD)based charge control techniques are used for distributed control of EV charging. Interestingly, the authors study the problem from the user perspective rather than the utility perspective; they consider various scenarios and objective functions, and propose a separate AIMD-like algorithm for each scenario. Our approach differs from their approach in four ways. First, our congestion control algorithm deals with a network in which a set of EVs may share multiple lines and transformers, whereas their control algorithm assumes a single resource with a certain available capacity shared by all EVs. Second, they study several scenarios with different objective functions; however,

\footnotetext{
${ }^{2}$ We believe that users have an incentive to lie about their deadlines to receive a higher charging rate if everyone pays the same.
} 
we focus on implementation and performance analysis of the control algorithm in a single scenario where the objective is to meet specific fairness and efficiency criteria. Third, we provide engineering guidelines to utilities for choosing control parameters and setpoints. Finally, our work is based on the theory of network utility maximization rather than an arbitrary choice of AIMD as the rate-control algorithm.

In [23] a control mechanism is designed to deal with the transformer overloading by modelling the transformer thermal limit as a constraint. Specifically, the authors formulate the EV charging problem as an open-loop centralized control problem with the objective of minimizing the SOC deviations from 1 and also minimizing the control effort subject to the capacity constraint of batteries and EV chargers, temperature constraint of the substation transformer, and the target SOC specified by EV owners. Using the dual decomposition method, an iterative price-coordinated implementation of this control mechanism is proposed which allows EV owners to compute their charging rate locally. To account for unexpected disturbances (i.e., fluctuations in the background demand), changing numbers of EVs, changing ambient temperature, and modelling errors they employ a receding-horizon feedback mechanism.

This work differs from ours in three ways. First, the proposed control method only deals with congestion at the substation transformer and does not address the overcapacity problem of feeders and other distribution transformers. Second, they do not consider fairness from the user's perspective. Third, they do not model the distribution network, and do not use power flow analysis to study the operation of the algorithm in a test distribution network.

Note that none of the works discussed above evaluates the proposed solution using power-flow analysis, as we do.

This work builds on our prior work in this area. The idea of real-time distributed control of the EV charging was first introduced in [13] and this paper uses the control architecture discussed in [12]. We also draw on the seminal work of Low and Lapsley on flow control [15]. The authors proved and evaluated using extensive simulations that a distributed price-based iterative algorithm that takes a control action in every iteration converges as long as changes are sufficiently small in every iteration.

\section{BACKGROUND AND ASSUMPTIONS}

We study a radial distribution network that supplies both uncontrolled (home) and controlled (EV) loads, as illustrated in Fig. 1. The topology of this network is encoded into a matrix $R$, where $R_{s l}$ is 1 if charger $s$ is downstream of line or transformer $l$, and is 0 otherwise.

\section{A. Nameplate Rating and Setpoint}

Every line or transformer in a distribution network has a nameplate rating. Equipment load must not exceed its nameplate rating over an extended period of time. We quantify distribution network congestion as the amount of energy transferred over and above the equipment's nameplate rating over a specified time period.

As part of our scheme, we allow a utility to associate a setpoint with every line or transformer. Our control goal is for the aggregate load (the sum of controlled and uncontrolled loads supplied by this equipment) to converge to this setpoint with

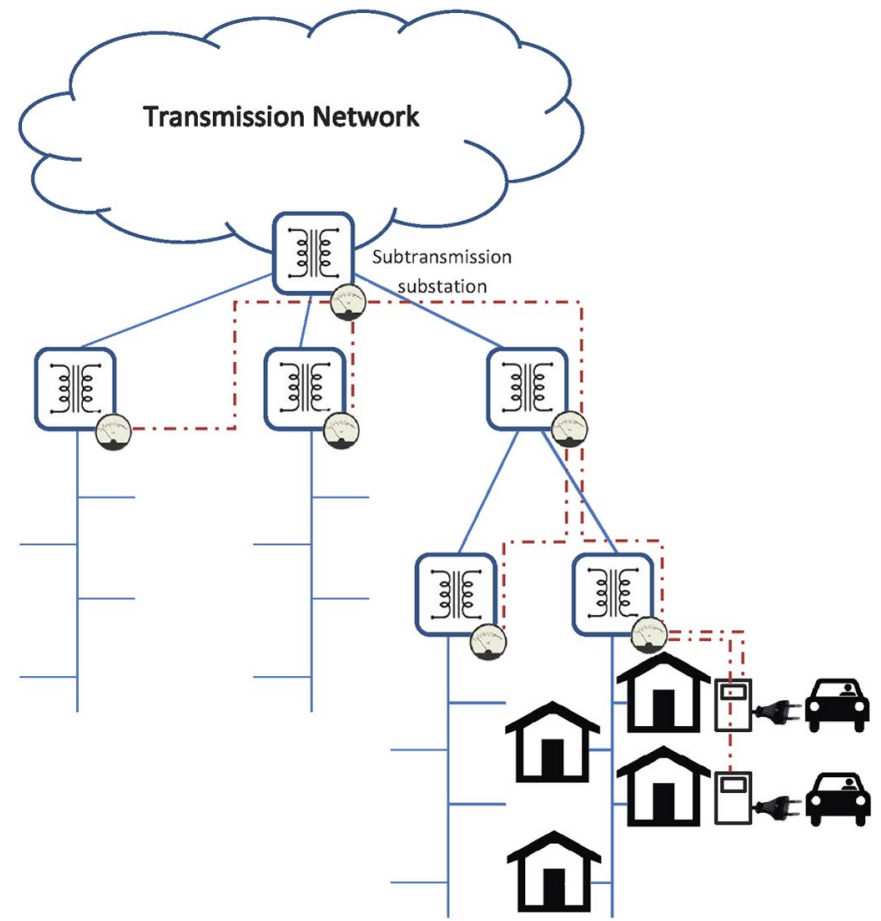

Fig. 1. An illustration of a smart distribution network consisting of MCC nodes, and communication links (dashed lines).

only limited congestion, i.e., a limited number of excursions above the nameplate rating. Thus, a conservative utility can ensure a very low congestion level by choosing an appropriately low setpoint.

\section{B. Measurement, Communication, and Control Nodes}

We assume that lines and transformers are supplemented by measurement, communication, and control (MCC) nodes [13]. MCC nodes play three key roles in our proposed solution. First, they continuously measure the congestion state of their corresponding line or transformer, where the congestion state of a line or transformer is defined as the difference between its setpoint and its current loading level. Second, they compute congestion prices in each signalling cycle (see Section III-D). Finally, they send these congestion prices to EV chargers downstream to allow them to independently choose their rates (see Section VI).

As shown in Fig. 1, the root MCC node is installed at the substation. It can send congestion signals on behalf of the (external) transmission and generation systems to reduce the EV charging load in response to generation shortfall or transmission network congestion. However, we do not consider these events in our work.

\section{Assumptions}

We now state the system assumptions that we make in developing our control algorithm.

A1) The communication network is ubiquitous, broadband, reliable, and has a low latency.

A2) MCC nodes can detect line or transformer overload sufficiently quickly that any transient overload is within system tolerances and the protection system is not invoked. This is true in nearly all distribution networks, 
where protection systems disconnect loads only when the overload is very large or persists for a long time.

A3) It is not possible to infer congestion implicitly at EV chargers. Therefore, congestion must be explicitly signalled to them.

A4) EVs only charge using EV chargers that are tamper-resistant and are under control of the electric utility. Thus, any control signal sent to them is assured of a cooperative response. ${ }^{3}$

A5) An EV battery can be charged at any rate in the range $\left[0, m_{s}\right]$, where $m_{s}$ is the maximum Amperage rating of its charger, independent of its state of charge. ${ }^{4}$

A6) The power factor is close to unity and therefore reactive power flow can be neglected. This allows us to use a simple DC system model in our work. Moreover, distribution system losses are assumed to be negligible. We relax both assumptions in our simulation studies in Section IX.

These assumptions imply that it is feasible to design and implement a control algorithm that changes the EV charging rate rapidly in response to the congestion state of the distribution system.

\section{System Operation}

We briefly sketch the operation of our system. Every $T_{c}$ milliseconds (the control timescale) the root MCC node initiates a signalling cycle by computing and sending its congestion price to its direct children. Upon receiving the congestion price(s), an intermediate MCC node computes and sends its own congestion price, using its latest recorded congestion state (as discussed in Section V-C1), along with the received price(s) to its children. Thus, EV chargers receive the set of congestion prices of all their parents. In Section V-C2, we explain how EV chargers use these congestion prices to choose their charging rate.

We now discuss the mathematical analysis used to obtain the congestion prices at each MCC node and the selection of charging rates at each EV charger.

\section{Optimization Problem}

In this section we formulate the control problem as a centralized static constrained optimization problem. The global objective function is chosen such that the solution to this optimization problem also guarantees proportional fairness.

Our objective is to allocate the available capacity of the network efficiently among active $\mathrm{EV}$ chargers without overloading the distribution network such that this allocation is fair to EV owners. We adopt the notion of proportional fairness which is an axiomatically justified fairness criterion [19]. It can be shown that proportional fairness is achieved if we maximize the value of a global objective function defined as the sum of the logarithm of the utility function of the users [18]. Since the departure of EVs from homes and charging stations is non-deterministic, it is reasonable to assume that $\mathrm{EV}$ owners are greedy and prefer to finish charging their EVs as soon as possible. Hence, the utility of a user $s$ is defined as the rate at which their EV is

\footnotetext{
${ }^{3}$ Note that our control algorithm would continue to operate if we assume cooperative response only from a subset of users; the uncooperative loads would act (and be considered) as uncontrolled home loads.

${ }^{4}$ With some battery technologies, the charging rate decreases as the state of charge increases. We do not consider this in our present analysis.
}

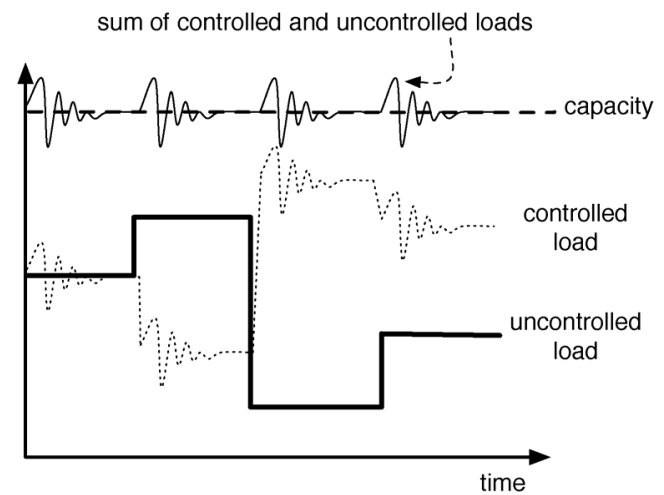

Fig. 2. The proposed control algorithm adapts charging rates of EV chargers to the available capacity of the network.

being charged, denoted $x_{s}$. For notational simplicity, we denote $\log \left(x_{s}\right)$ by $U_{s}\left(x_{s}\right)$. Observe that $U_{s}$ is infinitely differentiable, increasing, and strictly concave on its domain.

Our optimization problem is therefore a maximization of the sum of the $U_{s}$ of active chargers (i.e., those chargers that are charging an EV), subject to physical constraints imposed by chargers, lines, and transformers. The constraint which corresponds to each line or transformer is that its loading level cannot exceed its setpoint. However, since each line or transformer supplies both homes and EV chargers, ${ }^{5}$ and the aggregate home load imposed on it along with the set of active EV chargers supplied by it change over time, we decompose the problem into a series of snapshots, where, in each snapshot, home loads are constant and a fixed number of EVs are plugged in to chargers as shown in Fig. 2. We then formulate an optimization problem for each snapshot and derive control rules from that problem.

Consider the $n$th snapshot of the system in which the aggregate home load imposed on the line or transformer $l$ is denoted by $h_{l}^{n}$, and the set of active chargers is denoted by $\mathcal{S}^{n}$. The optimization problem is:

$$
\begin{aligned}
\max _{x} & \sum_{s \in \mathcal{S}^{n}} U_{s}\left(x_{s}\right) \\
\text { subject to } & 0 \leq x_{s} \leq m_{s} \quad \forall s \in \mathcal{S}^{n} \\
& y_{l} \leq \xi_{l}-h_{l}^{n} \quad \forall l \in \mathcal{L},
\end{aligned}
$$

where $\xi_{l}$ is the setpoint of $l, y_{l}$ is the total EV charging load imposed on $l$ (i.e., $y_{l}=\sum_{s: R_{s l}=1} x_{s}$ ), and $\mathcal{L}$ is the set of distribution lines and transformers equipped with MCC nodes. This problem is a convex optimization problem because it maximizes an objective function that is the sum of concave functions (and is therefore concave), and each constraint defines a convex set. Note that we refer to the second inequality constraint of (1) as the coupling constraint.

In the next section we obtain the dual problem and apply the dual decomposition method to obtain a set of decoupled subproblems. We then design a distributed algorithm that solves these subproblems locally and independently.

\section{Controller Design}

The centralized optimization problem formulated in the previous section can be solved using a distributed approach. The distributed approach has three key advantages over the centralized approach. First, it gives autonomy to local controllers

\footnotetext{
${ }^{5}$ For simplicity we ignore active power losses in the distribution network.
} 
thereby increasing robustness of the control system. Second, it is more scalable. Third, it decreases the overall latency of control because control decisions are made locally.

Our plan is, therefore, to design a distributed control algorithm by solving the Lagrangian dual of the centralized optimization problem. We apply the dual decomposition method to obtain a set of decoupled subproblems that are controlled at the higher level by a master problem through congestion prices. The proposed algorithm requires solving the master problem and these subproblems in an iterative fashion. From a control theory standpoint, solutions to these problems constitute our controls and congestion prices are the feedback.

\section{A. Dual Problem}

Consider the Lagrangian relaxation of the optimization problem (1):

$$
g(\lambda)=\max _{0 \preceq x \preceq m}\left\{\sum_{s \in \mathcal{S}} \log x_{s}+\sum_{l \in \mathcal{L}} \lambda_{l}\left(c_{l}^{n}-y_{l}\right)\right\},
$$

where $c_{l}^{n}=\xi_{l}-h_{l}^{n}$ denotes the available capacity of $l$ in the $n$th snapshot, $x=\left(x_{1}, \ldots, x_{|\mathcal{S}|}\right)$ is a vector of charging rates, $m=\left(m_{1}, \ldots, m_{|\mathcal{S}|}\right)$ is a vector of the charge capacity of EV chargers, $\lambda=\left(\lambda_{1}, \ldots, \lambda_{\mathcal{L} \mid}\right)$ is a vector of Lagrangian multipliers associated with the coupling constraints, and $\preceq$ is the vector inequality operator. Thus, the dual problem is

$$
\begin{aligned}
& \min _{\lambda} \max _{0 \preceq x \preceq m}\left\{\sum_{s \in \mathcal{S}} \log x_{s}+\sum_{l \in \mathcal{L}} \lambda_{l}\left(c_{l}^{n}-y_{l}\right)\right\} \\
& \text { subject to } \quad \lambda_{l} \geq 0 \quad \forall l \in \mathcal{L},
\end{aligned}
$$

which is equivalent to

$$
\begin{aligned}
& \min _{\lambda}\left\{\sum_{l \in \mathcal{L}} \lambda_{l} c_{l}^{n}+\max _{0 \preceq x \preceq m}\left\{\sum_{s \in \mathcal{S}} f_{s}\left(x_{s} ; \lambda\right)\right\}\right\} \\
& \text { subject to } \quad \lambda_{l} \geq 0 \quad \forall l \in \mathcal{L},
\end{aligned}
$$

where

$$
f_{s}\left(x_{s} ; \lambda\right)=\log x_{s}-x_{s} \sum_{l: R_{s l}=1} \lambda_{l}
$$

Note that we do not introduce dual variables for the constraints $0 \leq x_{s} \leq m_{s}$; hence, the maximization over $x$ is restricted to $0 \leq x_{s} \leq m_{s}$ for all values of $s$ (see [21, Sec. 3.4.2]). In the above equation, $f(x ; \lambda)$ represents $f$ as a function of $x$ parameterized by $\lambda$. Since $f_{s}\left(x_{s} ; \lambda\right)$ is the sum of two concave functions of $x_{s}$, it is also concave and has a unique maximum.

We note that (4) is derived from (3) by using the following equation.

$$
\sum_{l \in \mathcal{L}}\left(\lambda_{l} \sum_{s: R_{s l}=1} x_{s}\right)=\sum_{s \in \mathcal{S}}\left(x_{s} \sum_{l: R_{s l}=1} \lambda_{l}\right)=x R \lambda^{T}
$$

Importantly, in our formulation, strong duality holds because all inequality constraints are affine. Therefore, we can write the following KKT optimality conditions

$$
\hat{x}_{s}=\left[\frac{1}{\sum_{l: R_{s l}=1} \hat{\lambda}_{l}}\right]_{0}^{m_{s}} \quad \forall s \in \mathcal{S}
$$

$$
\begin{array}{lr}
\hat{\lambda}_{l}\left(\hat{y}_{l}-c_{l}^{n}\right)=0 & \forall l \in \mathcal{L} \\
\hat{y}_{l} \leq c_{l}^{n} & \forall l \in \mathcal{L} \\
0 \leq \hat{x}_{s} \leq m_{s} & \forall s \in \mathcal{S} \\
\hat{\lambda}_{l} \geq 0 & \forall l \in \mathcal{L}
\end{array}
$$

where $\hat{x}$ and $\hat{\lambda}$ are the unique optimizers of the Lagrangian dual problem. The first condition says that the gradient of Lagrangian vanishes at the optimal point, and the second condition, i.e., the complementary slackness condition, implies that either the optimal Lagrangian multiplier is zero, or the corresponding line or transformer is fully utilized, i.e., the line or transformer loading reached its nominal setpoint. Combining the first three conditions gives us the following relation between $\hat{x}$ and $\hat{\lambda}$.

$$
\hat{x}_{s}=\min \left\{\frac{1}{\sum_{l: R_{s l}=1} \hat{\lambda}_{l}}, m_{s}\right\}
$$

\section{B. Dual Decomposition}

Writing the Lagrangian dual problem in the form of (4) reveals its hidden decomposition structure [20]. Specifically, each EV charger can locally solve a subproblem given by

$$
\max _{0 \leq x_{s} \leq m_{s}} f_{s}\left(x_{s} ; \lambda\right)
$$

provided that it knows the sum of the Lagrangian multipliers corresponding to the lines and transformers that are supplying its load. It turns out that Lagrangian multipliers play the role of congestion prices (or shadow prices [14]) in our problem.

These subproblems are controlled by a master problem by means of congestion prices. The master problem is responsible for updating the congestion prices and can be written in the following form

$$
\min _{\lambda \succeq 0}\left\{\sum_{l \in \mathcal{L}} \lambda_{l} c_{l}^{n}+\sum_{s \in \mathcal{S}} f_{s}\left(\hat{x}_{s} ; \lambda\right)\right\} .
$$

where $f_{s}\left(\hat{x}_{s} ; \lambda\right)$ is the optimal value of (12). Observe that the objective function of the master problem is linear in $\lambda$ and its derivative with respect to a Lagrangian multiplier is given by

$$
\frac{\partial g}{\partial \lambda_{l}}(\lambda)=c_{l}^{n}-y_{l}
$$

\section{Control Laws}

Our approach is to solve the dual optimization problem using a distributed algorithm which has two separate parts. The first part adjusts congestion prices of lines and transformers by periodically measuring the available capacity and solving the master problem at each MCC using the gradient projection method. The second part updates the charging rates of EVs by solving the subproblems.

In the following we derive control laws for updating congestion prices and adjusting charging rates by solving the master problem and the subproblems respectively. These control laws constitute the distributed algorithm outlined in Section VI. In Section VII, we specify sufficient conditions for convergence of this algorithm to primal and dual optimal values. 
1) A Control Law for Updating the Congestion Price: Since the dual function is differentiable, we can adopt the gradient method with a projection onto the positive orthant to solve the master problem (13). The following algorithm updates congestion prices in each iteration in opposite direction to the gradient of the dual function.

$$
\lambda_{l}(t+1)=\max \left\{\lambda_{l}(t)-\kappa\left(c_{l}^{n}-y_{l}(t)\right), 0\right\} \quad \forall l \in \mathcal{L}
$$

Here $\kappa$ is a sufficiently small positive constant which determines the responsiveness and stability of control. Note that it is not necessary to estimate $c_{l}^{n}$ and $y_{l}$ separately at an MCC node to compute $c_{l}^{n}-y_{l}$. This is because $c_{l}^{n}-y_{l}$ is equal to the congestion state of $l$, i.e., the total line or transformer loading subtracted from its setpoint, and the congestion state is what being measured by the corresponding MCC node.

2) A Control Law for Adjusting the Charging Rate: We denote the latest congestion price vector that an EV charger has received by $\lambda(\bar{t})$. The subproblem (12) can be easily solved by finding the stationary point of $f_{s}\left(x_{s} ; \lambda\right)$.

$$
\begin{aligned}
f_{s}^{\prime}\left(x_{s}(t) ; \lambda(\bar{t})\right) & =\frac{1}{x_{s}(t)}-\sum_{l: R_{s l}=1} \lambda_{l}(\bar{t}) \stackrel{\text { set to }}{=} 0 \rightarrow \\
x_{s}(t) & =\min \left\{\frac{1}{\sum_{l: R_{s l}=1} \lambda_{l}(\bar{t})}, m_{s}\right\}
\end{aligned}
$$

Note that $x_{s}(t)$ is the rate of EV charger $s$ for the interval $[t, t+1)$, and adjusting the charging rates impacts the loading of upstream feeders and transformers immediately. ${ }^{6}$ More specifically, $y_{l}(t)$ is given by

$$
y_{l}(t)=\sum_{s: R_{s l}=1} x_{s}(t) \quad \forall l \in \mathcal{L}
$$

Note that the unit of time in (14) and (15) is $T_{c}$ milliseconds, and therefore $\bar{t}$ equals $t-\left(d / T_{c}\right)$ because congestion prices are received by EV chargers after $d$ milliseconds, which is an upper bound on the one-way latency from an MCC node and its downstream EV chargers.

\section{Distributed Charging Control Algorithm}

We now describe the algorithms that operate at the MCC nodes and at EV chargers and implement the control laws derived in Section $\mathrm{V}$.

Our distributed charging control algorithm measures the congestion state of a line or a transformer and computes the corresponding congestion price based on (14). This price is sent to descendant EV chargers every $T_{c}$ milliseconds (see Algorithm 1).

After receiving congestion prices from upstream MCC nodes, every charger computes its charging rate using (15) and starts charging at this rate (see Algorithm 2). Thus, it takes a control action every $T_{c}$ milliseconds using the most recent prices. In the next section, we show that this algorithm converges as long as some conditions are satisfied.

\footnotetext{
${ }^{6}$ There is a fundamental difference between congestion control protocols in the Internet and our EV charge control protocol. In computer networks, when traffic sources change their rates it is only reflected on link utilization after a delay, known as the forward delay. However, there is no forward delay in our problem as power flows in the grid at the speed of light.
}

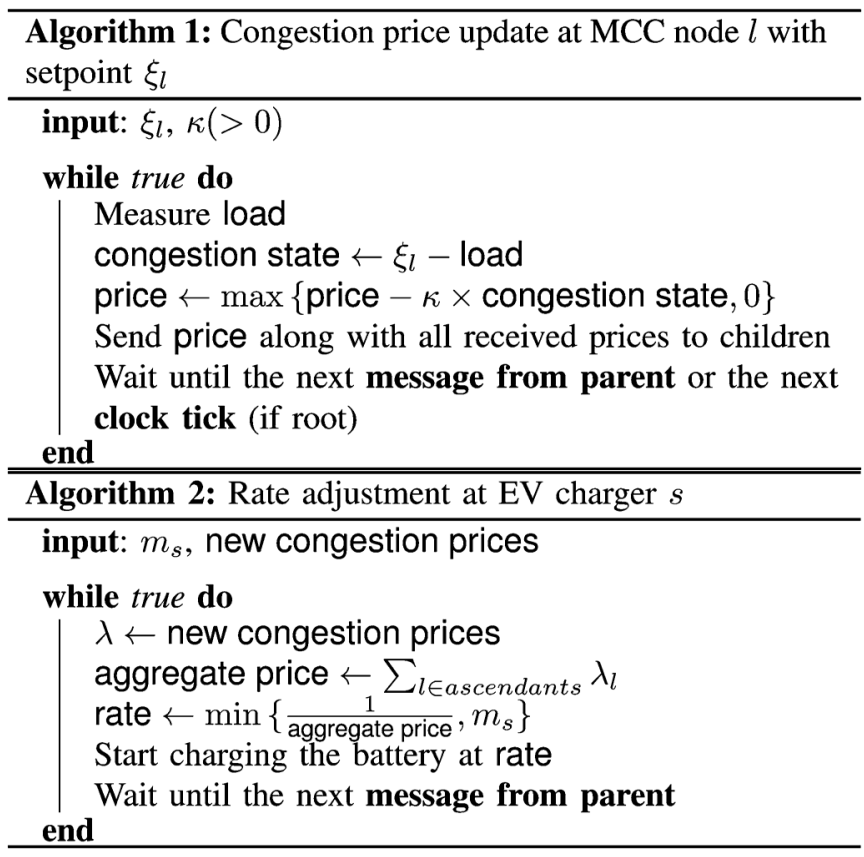

\section{CONVERGENCE ANALYSIS}

This section investigates the impact of control parameters (the gradient step size and the timescale of control) on the stability of the algorithm. We study the conditions under which the proposed distributed control algorithm converges to the solution of the centralized optimization problem (1) in a static setting, i.e., no EVs arrive or depart and the change in the magnitude of uncontrollable loads is negligible.

\section{A. Proof of Stability}

Note that the primal optimum is equal to the dual optimum as strong duality holds. Therefore, in this setting, we only need to show that the distributed control algorithm converges to the solution of (3). We then verify convergence in a dynamic setting both by studying the worst-case change in home loads, and through extensive numerical simulations.

Let $\bar{L}:=\max _{s} \sum_{l} R_{s l}$ be the length of the longest path from the substation to an EV charger, $\bar{S}:=\max _{l} \sum_{s} R_{s l}$ be the maximum number of active EV chargers sharing a link, $\bar{m}:=\max _{s} m_{s}$ be the maximum charging rate supported by EV chargers, and $d$ be the maximum communication delay between the root MCC node and any EV charger.

Theorem 1: Starting from any initial charging rates $0 \preceq x \preceq$ $m$ and congestion prices $\lambda \succeq 0$, the distributed control algorithm converges to the primal-dual optimal values if

1) $T_{c} \geq d$

2) $0<\kappa<\kappa^{*}=2 / \bar{m}^{2} \overline{L S}$

Proof sketch: The first condition guarantees that the control action at each MCC node, i.e., a change in congestion price, is taken only after all the EV chargers have reacted to the previous control action. In this case, the continuous time system reduces to the discrete-time system studied in [15] and our theorem reduces to Theorem 1 proved in that work. The second condition maps directly to the necessary condition for Theorem 1 in [15].

It can be proved that the control algorithm exhibits monotone convergence even in the worst case. This allows us to compute an upper bound on its convergence time. The proof of monotone 
TABLE I

The Total Home Load and the Number of EV Chargers Connected to a Specific Phase of a Load Bus in the Static Setting, and the Number of Homes and the Percentage of EV Population Connected to a Specific Phase of a Load Bus in the Dynamic Setting

\begin{tabular}{|c|c|c|c|c|c|c|c|c|c|c|c|c|c|c|c|c|c|c|}
\hline & Bus & \multicolumn{3}{|c|}{680} & \multicolumn{3}{|c|}{634} & \multicolumn{3}{|c|}{675} & \multicolumn{2}{|c|}{645} & \multicolumn{2}{|c|}{646} & \multicolumn{2}{|c|}{684} & 652 & 611 \\
\hline & Phase & $\mathrm{a}$ & $\mathrm{b}$ & $\mathrm{c}$ & $\mathrm{a}$ & $\mathrm{b}$ & $\mathrm{c}$ & $\mathrm{a}$ & $\mathrm{b}$ & $\mathrm{c}$ & $\mathrm{b}$ & $\mathrm{c}$ & $\mathrm{b}$ & $\mathrm{c}$ & $\mathrm{a}$ & $\mathrm{c}$ & $\mathrm{a}$ & $\mathrm{c}$ \\
\hline \multirow{2}{*}{ Static } & Agg. home load (kW) & 600 & 600 & 600 & 100 & 100 & 100 & 400 & 400 & 400 & 100 & 100 & 300 & 300 & 100 & 100 & 200 & 200 \\
\hline & Num of chargers & 80 & 80 & 80 & 40 & 40 & 40 & 40 & 40 & 40 & 40 & 40 & 40 & 40 & 40 & 40 & 40 & 40 \\
\hline \multirow{2}{*}{ Dynamic } & Num of homes & 450 & 450 & 450 & 50 & 50 & 50 & 300 & 300 & 300 & 50 & 50 & 200 & 200 & 50 & 50 & 150 & 150 \\
\hline & Percentage of chargers & $10 \%$ & $10 \%$ & $10 \%$ & $5 \%$ & $5 \%$ & $5 \%$ & $5 \%$ & $5 \%$ & $5 \%$ & $5 \%$ & $5 \%$ & $5 \%$ & $5 \%$ & $5 \%$ & $5 \%$ & $5 \%$ & $5 \%$ \\
\hline
\end{tabular}

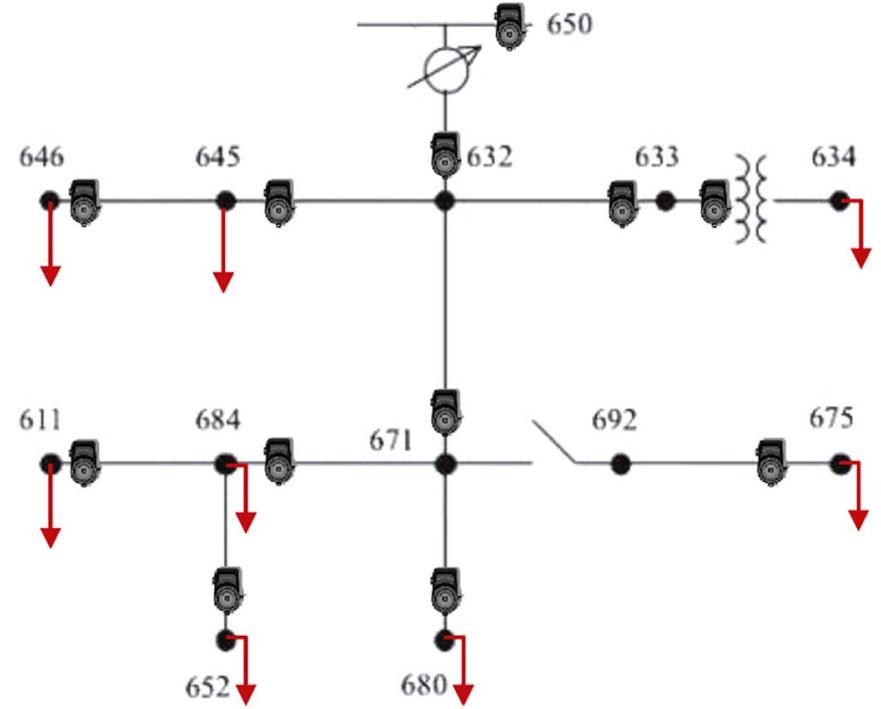

Fig. 3. The one-line diagram of our test distribution network. MCC nodes are shown as meters placed on the lines.

convergence and the upper bound on the convergence time are omitted due to space limitations and are available in [24].

\section{TEST Distribution SYSTEM}

We evaluate our control algorithm by means of power flow analysis using the Open Distribution System Simulator (OpenDSS) [25] on the standard IEEE 13-bus test feeder [26], a $4.16 \mathrm{kV}$ three-phase radial distribution system (Fig. 3). In this section, we discuss the details of this distribution network as well as our approach to model home and EV loads.

This distribution network is supplied by a three-phase 5 MVA transformer that steps down the transmission line voltage from $115 \mathrm{kV}$ to $4.16 \mathrm{kV}$. For sake of simplicity, we treat buses as load aggregation points with directly connected home loads and EV chargers and do not model the transformers and feeders radiating from them, although that analysis would be a straightforward adaptation to what we describe below. Using the parameters provided in [26], we set the nameplate rating of every feeder to its ampacity at $50^{\circ} \mathrm{C}$. The nameplate rating of the substation transformer and the in-line transformer are also set to $5 \mathrm{MVA}$ and $500 \mathrm{kVA}$ respectively. We assume that all loads, including homes and EV chargers, are single-phase and are connected between a phase and neutral of load buses $634,645,646,675,680$, $684,652,611$. We further assume that $\mathrm{EV}$ chargers are identical, either Level 1 (a maximum load of $1.8 \mathrm{~kW}$ ) or Level 2 (a maximum load of $7.2 \mathrm{~kW}$ ), consume real power only, and the reactive power consumption of every home is $30 \%$ of its real power consumption, a conservative assumption.

\section{A. MCC Nodes}

MCC nodes measure the load at a phase conductor on a load bus (Fig. 3). Similarly, the loading of the substation transformer and the in-line transformer, connected between buses 633 and 634, are also measured by an MCC node. All MCC nodes are interconnected using a communication network which forms a logical tree overlaid on the radial distribution network. Thus, for example the MCC node installed at phase B of bus 632 is the parent of MCC nodes installed at phase B of buses 633,645 , and 671 , and the MCC node installed at the substation is the parent of MCC nodes installed at phases A, B, and C of bus 632 .

\section{B. Power Flow}

To run a power flow study, we specify the amount of active and reactive power injected at every load bus. The OpenDSS simulator solves the optimization problem and provides the outputs power flows in different branches. Assuming that measurements from MCC nodes match the result of power flow calculations, we use these results in our control algorithm to obtain congestion prices. We compute the charging rate of EV chargers using the new prices sent by upstream MCC nodes, and update the aggregate EV charging load at every bus accordingly. We also update the aggregate home load at every bus using the synthetic load model described in Section VIII-C. This allows us to run a power flow study for the next iteration.

\section{Home Load Model}

We assume that our test distribution network supplies 3300 households, connected to load buses as described in Table I, and a finite population of EVs.

To evaluate our control algorithm using power flow and study its fast (sub-second) timescale dynamics, we need fine-grained measurements (100-millisecond timescale) of the household loads, which we lack. ${ }^{7}$ Therefore, we generate synthetic load traces using the Markov models developed in [27] for household electricity consumption during on-peak, mid-peak, and off-peak periods. These models are derived from fine-grained measurements of electricity consumption in 20 homes over four months. We then compute the aggregate home load imposed on the transformer by adding these loads, ignoring losses. Our simulations span over three days in winter and the corresponding aggregate home load is illustrated in Fig. 7. The peak of the aggregate home load is $4.44 \mathrm{MW}$ and hence the distribution system is never congested over these three days in the absence of EVs.

\footnotetext{
${ }^{7}$ We note that a real world implementation of the proposed algorithm only requires measurements of the branch power flow and transformer loading at the MCC nodes and does not rely on smart meter data or other measurements at the home level.
} 


\section{EV Model}

We assume that each household has at most one EV and a single EV charger that can charge only one EV at a time. We assume that the capacity of an EV battery is $24 \mathrm{kWh}$ (the capacity of a Nissan Leaf EV). We also assume that all EVs leave the system every day after 6 am following a Poisson distribution with parameter $\mu_{d}$, and return to the system after $4 \mathrm{pm}$ following a Poisson distribution with parameter $\mu_{a}$, with fully discharged batteries. Thus, the number of charging EVs changes with time. Since the EV population is finite, a higher value of $\mu_{a}\left(\right.$ or $\left.\mu_{d}\right)$ creates a larger burst of arrivals around $4 \mathrm{pm}$ (or departures around $6 \mathrm{am})$.

\section{Performance Evaluation}

This section uses power flow analysis to study:

- the effect of uncontrolled EV loads on a distribution network (Section IX-A),

- how long it takes for the distributed algorithm to converge to the chosen setpoint (Section IX-B),

- the dynamic behavior of our system (Section IX-C), and

- the efficiency of our control system, compared to the best possible efficiency (Section IX-D),

- the operation of the congestion control algorithm with a realistic number of MCC nodes (Section IX-E).

We measure the efficiency by the average energy stored in an EV battery in a day. We measure the congestion of a line or a transformer by the amount of energy it supplied when it is above its nameplate rating level (we refer to this as the overload below).

\section{A. The Need for Control}

Fig. 4 shows the effect of uncontrolled EV loads on the distribution network using Level 2 chargers. We find that a population of merely 90 EVs in a neighborhood comprised of 3300 homes leads to a non-negligible overload of $54 \mathrm{kWh} /$ day. To avoid congestion without controlled charging, either the EV penetration level must be kept low or lines and transformers must be upgraded. For example, if the utility requires an overload of less than $1 \mathrm{kWh} /$ day, depicted by a horizontal line in Fig. 4, without controlled charging, the EV population must be kept below 70 or $2.1 \%$. Even when the overall penetration level is low, this may not be true for certain neighborhoods.

\section{B. Rate of Convergence}

We now study the number of iterations that it takes to achieve convergence for different values of $\kappa$, assuming that the setpoint of each line or transformer is set to be $95 \%$ of its nameplate rating, that $\mathrm{EV}$ chargers charge at Level 1, and that the home loads do not change. Table I summarizes our simulation scenario in this static setting.

In this scenario, the maximum charging rate is $1800 \mathrm{~W}$, so the maximum step size for which the convergence of the algorithm is guaranteed is $\kappa^{*}=2 / 1800^{2} \times 800 \times 5=1.54 \times 10^{-10}$ (from Theorem 1). As we increase the value of $\kappa$, the control system transitions from an over-damped system to an under-damped system and eventually to an unstable system for $\kappa>3.8 \times 10^{-9}$ which is larger than $\kappa^{*}$. Fig. 5 shows how the loading of the substation transformer varies over different iterations for three

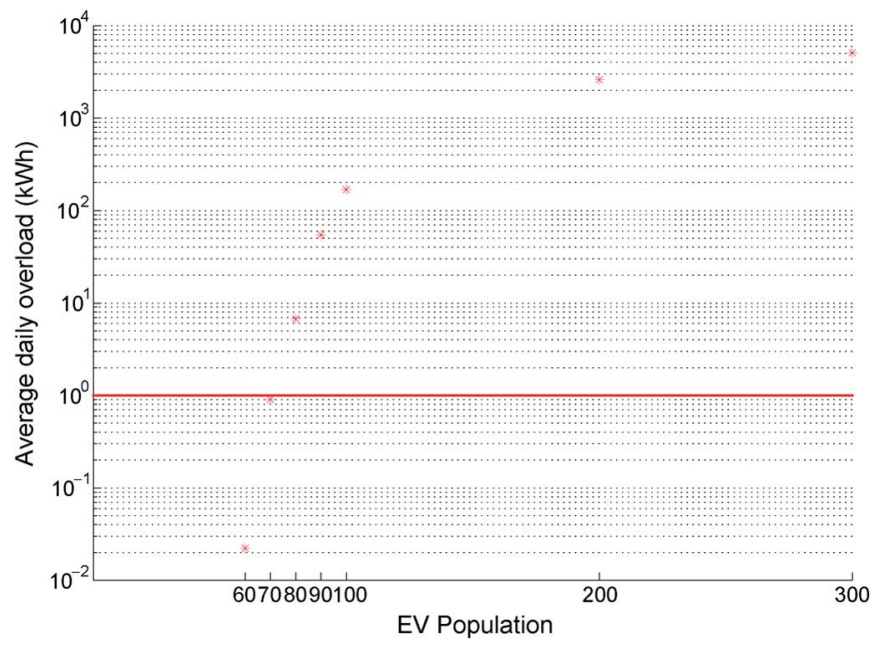

Fig. 4. Average daily overload versus EV population when EVs are charged by AC Level 2 chargers without control. Note that the Y-axis is logarithmic scale.

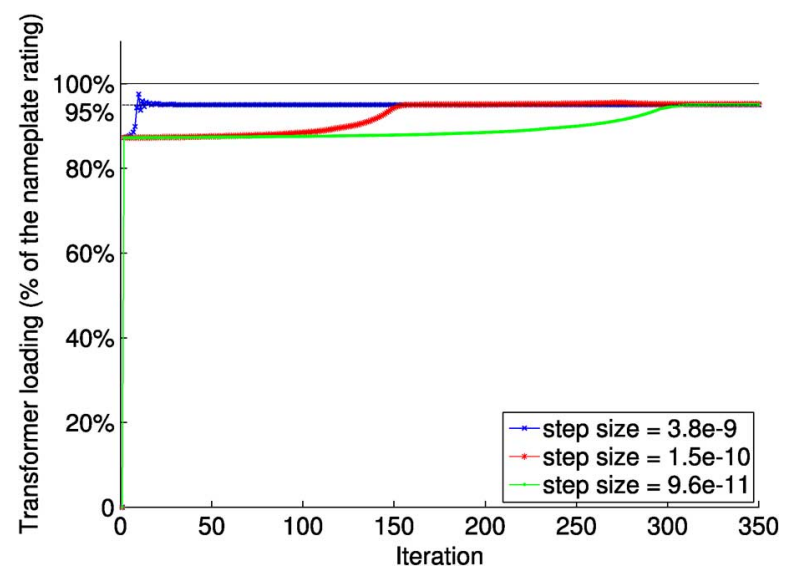

Fig. 5. The value of the step size $\kappa$ determines how the loading of the substation transformer changes over time. Here the equipment setpoint is set to $95 \%$ of its nameplate rating.

different values of $\kappa$ that are smaller than, equal to, and larger than $\kappa^{*}$.

The value of $\kappa$ also controls the number of iterations it takes to achieve convergence, that is, when the loading of a line or transformer is within $\pm 1 \%$ of its setpoint. Fig. 6 shows that the number of iterations required for convergence deceases exponentially as we increase the value of $\kappa$. When the step size is equal to $\kappa^{*}$, it takes 149 iterations to achieve convergence (about 15 seconds if the control timescale is chosen to be $0.1 \mathrm{~s})$. Note that in most cases the algorithm converges to $\pm 5 \%$ of the setpoint after only $20-40$ iterations.

\section{Performance Evaluation in a Dynamic Setting}

We now investigate the scenario when the home loads and the number of EVs are time-varying, as discussed in Section VIII. In these experiments, we fix the value of the step size to be $\kappa^{*}$, and study six different values for the setpoint of the substation transformer: 4.75 MVA, 4.8 MVA, 4.85 MVA, 4.9 MVA, 4.95 MVA, and 5 MVA, two different control timescales: 1 second, and 0.5 second, and two possible charging levels: AC Level 1 (1.8 kW maximum) and AC Level 2 (7.2 kW maximum) [28]. We repeat each simulation 10 times, using 10 different arrival 


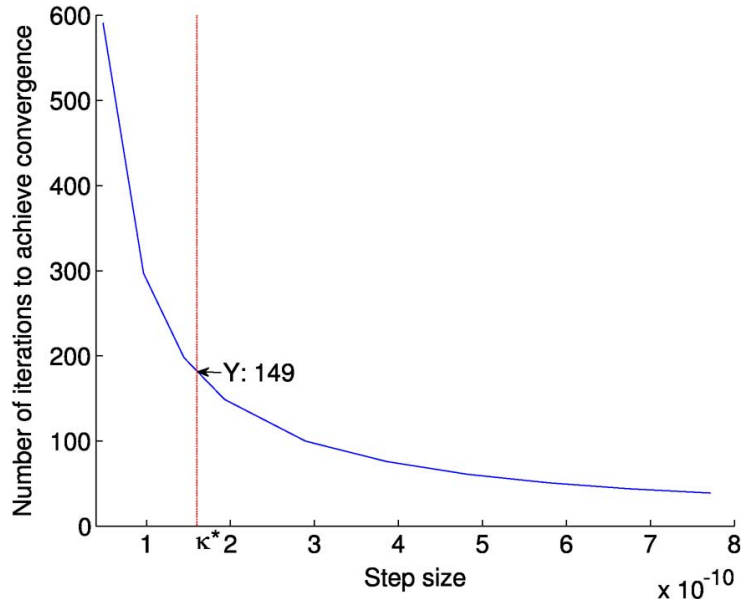

Fig. 6. The number of iterations to achieve convergence.

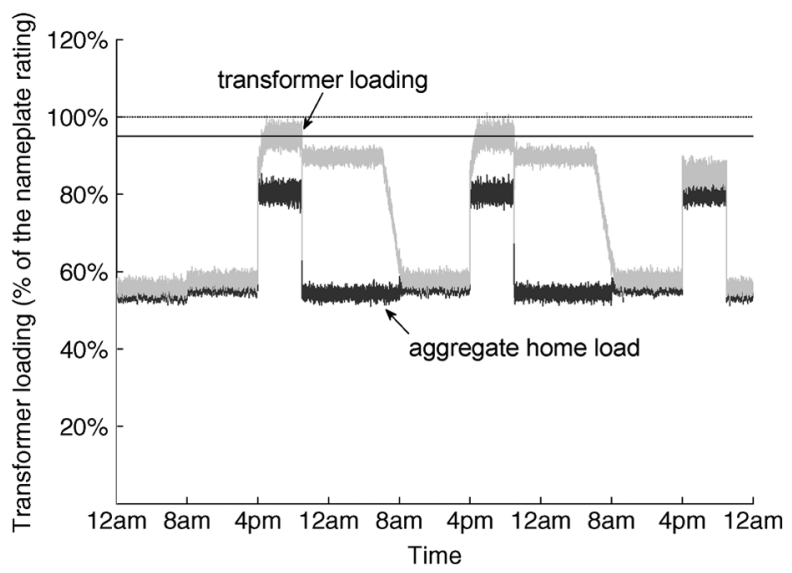

Fig. 7. Operation of the algorithm in a dynamic setting with 900 Level $1 \mathrm{EV}$ chargers where the substation transformer's setpoint is 4.75 MVA (the solid line) and the control timescale is 1 second. The aggregate home load is shown in dark grey and the overall load measured at the substation transformer in light grey.

and departure times generated by setting $\mu_{d}=\mu_{a}=0.1$ per second. ${ }^{8}$

Fig. 7 shows a single simulation trace of the overall load over time when the transformer setpoint is 4.75 MVA, the control timescale is 1 second, and the EV population is 900 . The home loads have evening peaks, with loads at night and at mid-day being roughly equal. Note that even when EVs are not charging, the transformer load is slightly higher than the aggregate home load due to line losses. When EVs are present, the overall load is close to the setpoint, with rare excursions above the nameplate rating. Each such excursion contributes to the overload. Clearly, the lower the setpoint, the lower this overload. To demonstrate this, Fig. 8 shows the average daily overload versus the transformer's setpoint when the EV population is 500. It can be readily seen that the overload decreases with lower setpoints, but increases as we use slower control timescales. It also increases significantly with the charging level, and grows exponentially with the setpoint for a specific control timescale and a specific charging level.

${ }^{8}$ Thus, on average, one EV arrives and departs every 10 seconds after 4 pm and 6 am respectively. These arrival and departure patterns are intentionally chosen to stress test the control algorithm.

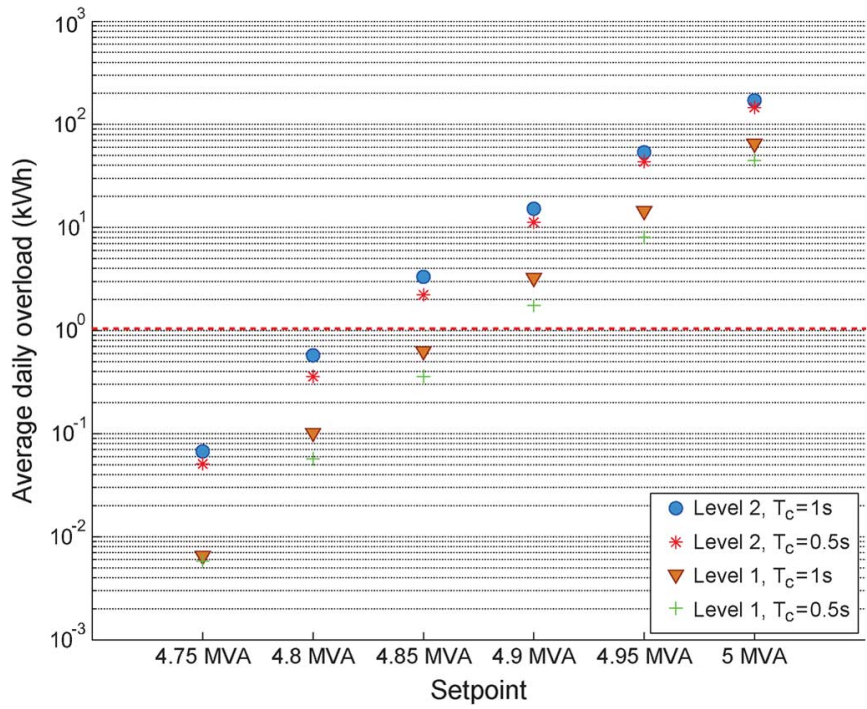

Fig. 8. Average daily overload versus setpoint when the EV population is 500 . Note that the Y-axis is logarithmic scale.

\section{Efficiency}

We now study the efficiency of our control scheme. Consider the situation in which all EVs arrive at $4 \mathrm{pm}$ and stay in the system until 6 am of the next day. We numerically compute an upper bound on the number of EVs that can be fully charged in this time interval by simply dividing the integral of the difference between the nameplate rating and the aggregate home load (after incorporating losses) over this interval by the battery capacity (i.e., $24 \mathrm{kWh}$ ). We find that a maximum of $900 \mathrm{EVs}$ can be fully charged between $4 \mathrm{pm}$ and 6 am of the next day under these ideal conditions. With our control algorithm, using Level 2 charging, if we set the setpoint of the substation transformer to 4.8 MVA to obtain a very small overload (approximately 1 $\mathrm{kWh}$ /day), up to around $700 \mathrm{EVs}$ can be fully charged which compares well with the maximum of $900 \mathrm{EVs}$ especially when recalling that without control we cannot charge more than 70 EVs to obtain the same level of overload.

\section{E. Impact of Partial Deployment of MCC Nodes}

The test distribution system described in Section VIII is a small network thoroughly monitored by the MCC nodes installed at the substation, distribution transformers, and buses. However, a real distribution network could have thousands of line segments and monitoring the power flow in every part of the network requires widespread deployment of MCC nodes, which is quite costly and impractical at the present time. This motivates us to study the operation of the congestion control mechanism with a realistic number of MCC nodes.

We argue that MCC nodes should be installed wherever congestion is likely to happen as congestion can only be detected locally. In reality, congestion is not likely to happen in all parts of a distribution network and the network has a certain number of hotspots. We call hotspot a line or a transformer that is i) susceptible to overload, i.e., the margin between its loading and its nameplate rating is small and ii) anticipated to supply a large number of EVs. We expect the number of hotspots to be relatively small (especially at low EV penetration rates) and the 
utilities to know or predict their locations. Hence, it is reasonable to install MCC nodes at these locations.

Consider the following cases where MCC nodes are installed 1) only at hotspots, 2) at hotspots and buses on the path from the substation to the hotspots, and 3) everywhere as illustrated in Fig. 3. We create a hotspot in our test distribution system by making a slight change to the dynamic scenario presented in Table I and compare these three cases based on the amount of overload. Specifically, we increase the number of homes supplied by phase B of bus 646 to 300, and reduce the number of homes supplied by each phase of bus 680 to 150 . This creates a single hotspot, i.e., phase B of the line segment between buses 645 and 632 becomes congested when all EV chargers are Level 1 and 500 EVs are uniformly distributed in the network. We choose the setpoint of all lines and transformers to be $98 \%$ of their nameplate rating. Results of power flow simulations show that the last two cases are similar in terms of the average daily overload $(\sim 20.35 \mathrm{kWh})$, while the first case leads to a slightly higher daily overload of $20.46 \mathrm{kWh}$. This indicates that the benefit from installing MCC nodes at non-hotspot locations is insignificant and our scheme does not necessitate a pervasive MCC deployment.

\section{ENGINEERING INSIGHTS}

This section provides guidelines for choosing the control parameters and setpoints based on the results of our simulations, assuming that the utility limits the amount of risk that is willing to take. For simplicity, we confine our study to the substation transformer which is the potential bottleneck in this network, although our study applies to arbitrary multi-level distribution networks. Thus, we only have one setpoint to select.

\section{A. Choosing Control Knobs: $\kappa$ and $T_{c}$}

Recall that the gradient step size, $\kappa$, should be set to $\kappa^{*}$ to improve responsiveness of control and ensure stability.

Choosing $T_{c}$ is more complex. Faster control timescales reduce the overload but increase the communication overhead. If communication is not a bottleneck, then $T_{c}$ should be set as small as possible. Of course, this is lower bounded by the communication delay, as stated by Theorem 1 .

Communication overhead can be reduced by using multicast to send packets from an MCC node to its children. With multicasting we can implement the control algorithm by sending at most $\mathcal{O}=|\mathcal{S}|+|\mathcal{L}|-1$ packets in every control interval, where $|\mathcal{S}|$ is the number of EV chargers and $|\mathcal{L}|$ is the number of MCC nodes. Hence, $\mathcal{O}, 2 \times \mathcal{O}$, and $10 \times \mathcal{O}$ packets must be transmitted per second when the control timescale is 1 second, 0.5 second, and 0.1 second respectively. Thus the control timescale can be chosen properly given the communication medium and protocol.

\section{B. Choosing Setpoints}

Recall that transformer overloading increases the risk of equipment failure and outages. The choice of setpoints of lines and transformers depends on the amount of risk the utility is willing to take. Specifically, the utility chooses the equipment setpoint for a given EV population and a charging level such that the overload does not exceed an acceptable level.

For example, in the above scenario (where we have only one setpoint to select) the setpoint of the transformer could be as

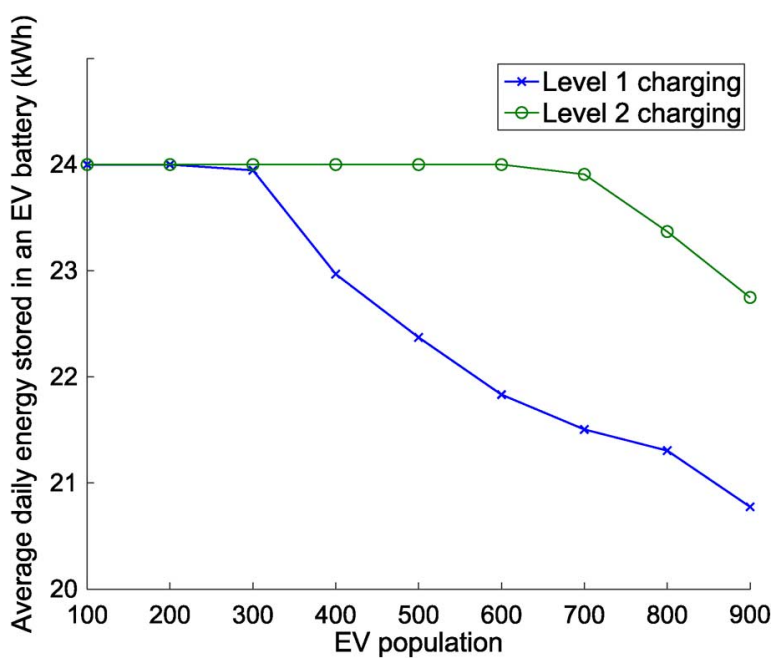

Fig. 9. Average energy stored in an EV battery in a day versus the EV population for AC Level 1 and Level 2 chargers when the control timescale is 1 second.

high as 4.85 MVA to obtain an overload of $1 \mathrm{kWh} /$ day (depicted by a dashed line in Fig. 8) when the EV population is 500 and all EV chargers are Level 1.

Fig. 9 shows the average daily energy stored in an EV battery for different EV population sizes using Level 1 and Level 2 chargers when the setpoint is chosen to obtain an overload of less than $1 \mathrm{kWh} /$ day, and the control timescale is set to 1 second. Observe that EV batteries are fully charged using both Level 1 and Level 2 chargers when the EV population is less than 300 (Level 2 charging only reduces the charging time). However, when the EV population exceeds 300 , the system becomes overly congested; in this regime, Level 2 charging is more beneficial to EV owners than Level 1 charging as it increases the efficiency. For example, when the EV population is 500 and all chargers are Level 2, the setpoint could be as high as 4.8 MVA; this corresponds to on average $24 \mathrm{kWh}$ of energy transferred to an EV per day, which means that all batteries can be fully charged. Similarly, when the EV population is 500 and all chargers are Level 1, the setpoint could be as high as 4.85 MVA; this corresponds to on average $22.37 \mathrm{kWh}$ of energy transferred to an EV per day.

\section{CONCLUSION}

Our work addresses line and transformer congestion arising from uncontrolled charging of electric vehicles. Motivated by rate control in computer networks, we propose a real-time, distributed, stable, efficient, and fair charging algorithm based on the dual-decomposition approach. This algorithm scales well with the size of the network and the number of EVs. We show through extensive numerical simulations as well as power flow analysis on a test distribution network that this algorithm operates successfully in both static and dynamic settings, despite changes in home loads and the number of connected EVs. We analyze the sensitivity of this algorithm to the EV penetration level, the rated charge capacity of EV chargers, the choice of control parameters, and control setpoints. Based on this analysis, we provide guidelines for choosing these parameters in a distribution network. 
Our work suffers from some limitations. The chief limitation is that it requires the installation of MCC nodes at all potential points of congestion in the distribution network. A second problem is that our control algorithm does not scale particularly well: the choice of $\kappa$, which controls system responsiveness, is upper-bounded by the number of EVs and the maximum EV charging rate. As these increase, $\kappa$ and system responsiveness decrease. What is needed is a less conservative bound for $\kappa$ that has better scaling properties. Third, our EV battery model is simple and ignores the relationship between the charging rate and the state-of-charge of the battery. Finally, our approach does not deal with other consequences of EV charging including large voltage swings and phase imbalance. We plan to address these limitations in future work.

\section{REFERENCES}

[1] J. Lopes, F. Soares, and P. Almeida, "Integration of electric vehicles in the electric power system," Proc. IEEE, vol. 99, no. 1, pp. 168-183, 2011.

[2] S. Shao, M. Pipattanasomporn, and S. Rahman, "Challenges of PHEV penetration to the residential distribution network," in Proc. IEEE Power Energy Soc. Gen. Meet., 2009, pp. 1-8.

[3] C. Ahn, C.-T. Li, and H. Peng, "Optimal decentralized charging control algorithm for electrified vehicles connected to smart grid," J. Power Sources, vol. 196, no. 2, pp. 10 369-10 379, 2011.

[4] E. Sortomme, M. Hindi, S. MacPherson, and S. Venkata, "Coordinated charging of plug-in hybrid electric vehicles to minimize distribution system losses," IEEE Trans. Smart Grid, vol. 2, no. 1, pp. 198-205, 2011.

[5] K. Clement-Nyns, E. Haesen, and J. Driesen, "The impact of charging plug-in hybrid electric vehicles on a residential distribution grid," IEEE Trans. Power Systems, vol. 25, no. 1, pp. 371-380, 2010.

[6] Z. Ma, D. Callaway, and I. Hiskens, "Decentralized charging control of large populations of plug-in electric vehicles," IEEE Trans. Control Syst. Technol., vol. 21, no. 1, pp. 67-78, 2013.

[7] L. Gan, U. Topcu, and S. Low, "Optimal decentralized protocol for electric vehicle charging," IEEE Trans. Power Syst., vol. 28, no. 2, pp. 940-951, 2013.

[8] S. Deilami, A. Masoum, P. Moses, and M. A. S. Masoum, "Real-time coordination of plug-in electric vehicle charging in smart grids to minimize power losses and improve voltage profile," IEEE Trans. Smart Grid, vol. 2, no. 3, pp. 456-467, 2011.

[9] S. Studli, E. Crisostomi, R. Middleton, and R. Shorten, "Aimd-like algorithms for charging electric and plug-in hybrid vehicles," in Proc. IEEE Int. Elect. Veh. Conf. (IEVC), 2012, pp. 1-8.

[10] C.-K. Wen, J.-C. Chen, J.-H. Teng, and P. Ting, "Decentralized plug-in electric vehicle charging selection algorithm in power systems," IEEE Trans. Smart Grid, vol. 3, no. 4, pp. 1779-1789, 2012.

[11] Z. Fan, "A distributed demand response algorithm and its application to phev charging in smart grids," IEEE Trans. Smart Grid, vol. 3, no. 3, pp. 1280-1290, 2012.

[12] O. Ardakanian, C. Rosenberg, and S. Keshav, "Distributed control of electric vehicle charging," in Proc. e-Energy'13 ACM, pp. 101-112.

[13] O. Ardakanian, C. Rosenberg, and S. Keshav, "Realtime distributed congestion control for electrical vehicle charging," SIGMETRICS Perform. Eval. Rev., vol. 40, no. 3, pp. 38-42, Jan. 2012.
[14] F. P. Kelly, A. K. Maulloo, and D. K. H. Tan, "Rate control for communication networks: Shadow prices, proportional fairness and stability," J. Oper. Res. Soc., vol. 49, no. 3, pp. 237-252, 1998.

[15] S. H. Low and D. E. Lapsley, "Optimization flow control. I. Basic algorithm and convergence," IEEE/ACM Trans. Netw., vol. 7, no. 6, pp. 861-874, 1999.

[16] R. Srikant, The Mathematics of Internet Congestion Control (Systems and Control: Foundations and Applications). Boston, MA, USA: Birkhauser, 2004.

[17] F. Paganini, Z. Wang, J. Doyle, and S. Low, "Congestion control for high performance, stability, and fairness in general networks," IEEE/ACM Trans. Netw., vol. 13, no. 1, pp. 43-56, 2005.

[18] F. Kelly, "Charging and rate control for elastic traffic," Eur. Trans. Telecommun., vol. 8, no. 1, pp. 33-37, 1997.

[19] H. Yaïche, R. R. Mazumdar, and C. Rosenberg, "A game theoretic framework for bandwidth allocation and pricing in broadband networks," IEEE/ACM Trans. Netw., vol. 8, no. 5, pp. 667-678, 2000.

[20] D. Palomar and M. Chiang, "A tutorial on decomposition methods for network utility maximization," IEEE J. Sel. Areas Commun., vol. 24, no. 8, pp. 1439-1451, 2006.

[21] D. P. Bertsekas and J. N. Tsitsiklis, Parallel and Distributed Computation: Numerical Methods. Upper Saddle River, NJ, USA: Prentice-Hall, 1989.

[22] J. Taft and P. De Martini, "Cisco Systems-Ultra large-scale power system control architecture" [Online]. Available: http://www.cisco. $\mathrm{com} / \mathrm{web} /$ strategy/docs/energy/control_architecture.pdf

[23] R. Hermans, M. Almassalkhi, and I. Hiskens, "Incentive-based coordinated charging control of plug-in electric vehicles at the distribution-transformer level," in Proc. Amer. Control Conf. (ACC) 2012, pp. 264-269.

[24] O. Ardakanian, S. Keshav, and C. Rosenberg, "Real-time distributed control for smart electric vehicle chargers: From a static to a dynamic study," Univ. Waterloo, Waterloo, ON, Canada, Tech. Rep. CS-2013-20, Dec. 2013.

[25] EPRI, Simulation Tool-OpenDSS [Online]. Available: http://www. smartgrid.epri.com/SimulationTool.aspx

[26] W. Kersting, "Radial distribution test feeders," in Proc. IEEE Power Eng. Soc. Winter Meet. 2001, vol. 2, pp. 908-912.

[27] O. Ardakanian, S. Keshav, and C. Rosenberg, "Markovian models for home electricity consumption," in Proc. ACM SIGCOMM Green Netw. Workshop, 2011.

[28] SAE J1772 [Online]. Available: http://www.sae.org/smartgrid/chargingspeeds.pdf

Omid Ardakanian is currently a Ph.D. student in computer science at the University of Waterloo, Canada, and a member of the ISS4E research group. More information can be found at http://cs.uwaterloo.ca/ oardakan/.

Srinivasan Keshav is a Professor, ACM Fellow, Canada Research Chair, and Cisco Systems Research Chair in Smart Grid with the Cheriton School of Computer Science, University of Waterloo, Canada. More information can be found at http://cs.uwaterloo.ca/ keshav/.

Catherine Rosenberg ( $\left.F^{\prime} 11\right)$ is a Professor at the University of Waterloo, Canada, and holds a Tier 1 Canada Research Chair in the Future Internet. She is a Fellow of the Canadian Academy of Engineering. More information can be found at http://ece.uwaterloo.ca/ cath/. 